\title{
Combination Therapy with Pegylated Interferon Plus Ribavirin in the Treatment of Hepatitis C Virus-Related Thrombocytopenia
}

Tarkan Karakan, MD; Mehmet Cindoruk, MD; Bulent Degertekin, MD; Ibrahim Dogan, MD; Alper Sancak, MD; Sukru Dumlu, MD; Ahmet Gorgul, MD; and Selahattin Unal, MD

Gastroenterology Department, Gazi University, Faculty of Medicine, Ankara, Turkey

\section{ABSTRACT}

Background: Isolated thrombocytopenia is a common manifestation of hepatitis $\mathrm{C}$ virus (HCV) infection. There is no established treatment modality for this condition. The efficacy of standard interferon (IFN) monotherapy has been reported in some studies. The major disadvantage of this treatment is the high rate of recurrence due to viral breakthrough during the first 12 weeks of treatment. Pegylated IFNs are now the standard regimen for chronic hepatic disease due to HCV infection. However, due to a lack of evidence, pegylated IFNs are not widely used for HCV-related isolated thrombocytopenia.

Objective: The aim of this report was to present the case of a male patient with severe symptomatic thrombocytopenia due to HCV infection.

Methods: Thrombocytopenia was treated with pegylated IFN plus ribavirin.

Results: Although standard IFN monotherapy failed to achieve virologic and hematologic improvement, therapy with pegylated IFN alfa-2a plus ribavirin was associated with both virologic and hematologic improvement without any significant adverse effects.

Conclusions: Pegylated IFN plus ribavirin was effective in this patient for the treatment of HCV-related thrombocytopenia. However, further research is needed to define the response rate in different patient populations. (Curr Ther Res Clin Exp. 2005;66:247-253) Copyright (C 2005 Excerpta Medica, Inc.

Key words: hepatitis $\mathrm{C}$ virus, thrombocytopenia, pegylated interferon, ribavirin combination, case report. 


\section{INTRODUCTION}

Extrahepatic manifestations of hepatitis $\mathrm{C}$ virus (HCV) infection are observed in $\sim 38 \%$ to $50 \%$ of cases ${ }^{1}$; one of these manifestations is mild to moderate thrombocytopenia. Hepatic disease or cirrhosis with portal hypertension commonly leads to hypersplenism-related thrombocytopenia. Nonetheless, thrombocytopenia is more severe in HCV infections than in other hepatic diseases, possibly because of increased autoimmunity related to HCV infection. In HCV-related thrombocytopenia and idiopathic thrombocytopenic purpura (ITP), large thrombocytes are seen on peripheral smears, and a normal or elevated megakaryocyte count is seen in bone marrow aspirates, indicating peripheral destruction of thrombocytes.

Interferons (IFNs) are used in clinical practice for the treatment of HCV infection. ${ }^{1}$ Standard IFN monotherapy has been reported in some studies. ${ }^{1}$ Although the main effect of IFNs on HCV is the suppression of replication, ${ }^{1}$ other beneficial effects include immunomodulation of the host immune system. However, the major disadvantage of this treatment is the high rate of recurrence due to viral breakthrough during the first 12 weeks of treatment. ${ }^{2}$ In addition, few studies have reported improvement in HCV-related thrombocytopenia with IFN therapy, ${ }^{3}$ and current guidelines ${ }^{4}$ do not mention the treatment of HCV-related thrombocytopenia with IFNs. Pegylated IFNs are now the standard regimen for chronic hepatic disease due to HCV infection. However, due to a lack of evidence, pegylated IFNs are not widely used for HCV-related isolated thrombocytopenia.

Herein we report a case of HCV-related severe thrombocytopenia treated with IFN alfa-2a, with a fluctuating response. The patient provided written consent to use this material for publication.

\section{CASE PRESENTATION}

An 18-year-old man was admitted to Gazi University, Ankara, Turkey, for treatment of epistaxis. He had no history of alcohol consumption, use of medications or herbal remedies, or drug abuse. Physical examination, including vital signs, was unremarkable. Complete blood count revealed severe thrombocytopenia $(10,000$ cells $/ \mathrm{mL})$, and blood chemistry showed mildly elevated hepatic enzyme levels (alanine aminotransferase [ALT], $65 \mathrm{IU} / \mathrm{mL}$; aspartate aminotransferase [AST], $56 \mathrm{IU} / \mathrm{mL}$ [normal range for both, $0-40 \mathrm{IU} / \mathrm{mL}$ ]). The remainder of the blood chemistry results were normal, as were prothrombin time, activated partial thromboplastin time, bleeding time, antinuclear antibody test results, anticardiolipin antibodies (immunoglobulin [Ig] M, G, and A), platelet-associated antibodies (IgG and IgM), and thyroid function test results. The patient had no signs of cirrhosis, including peripheral edema, ascites, spider angiomas, gynecomastia, or palmar erythema. Hepatic function tests, including synthetic functions (eg, albumin, prothrombin time, activated partial thromboplastin time) and excretory functions (eg, total/conjugated bilirubin), were all within normal limits. Serologic markers for viral hepatitis and HIV were negative, 
except for HCV antibody. HCV reverse transcriptase polymerase chain reaction was $3 \times 10^{6}$ copies $/ \mathrm{mL}$. Genetic analysis revealed genotype $1 \mathrm{~b}$. Abdominal ultrasonography was unremarkable. Serum cryoglobulin studies were negative, and no symptoms of arthralgia or palpable purpura or signs of systemic vasculitis suggestive of cryoglobulinemia were present.

Physical examination revealed no lymphoid enlargement suggestive of malignant lymphoma. Peripheral blood smear revealed no abnormalities in myeloid or lymphoid cell lines. To rule out hereditary and other rare causes of thrombocytopenia, the bone marrow was aspirated. ${ }^{4}$ The aspirate showed a moderately elevated megakaryocyte count without any other abnormal cell infiltration, which would have been indicative of accelerated platelet destruction. The differential diagnosis included immunologic (drugs, idiopathic) and nonimmunologic (sepsis, disseminated intravascular coagulation, vasculitides, cardiac prosthesis) causes. Abnormal vessels, fibrin thrombi (sepsis, disseminated intravascular coagulation), and intravascular prostheses can all shorten platelet survival and cause nonimmunologic thrombocytopenia. However, the patient had no signs of vasculitis or vascular or cardiac prosthesis; serum fibrinogen and fibrin degradation products were normal. The other pathogenetic mechanism of accelerated thrombocytopenia is the immunologic destruction of platelets. The most common causes of immunologic thrombocytopenia are viral or bacterial infection, drug use (often heparin), and ITP. Patients with immunologic thrombocytopenia typically do not have splenomegaly, although they typically do have an elevated bone marrow megakaryocyte count, as did this patient.

As a result, we diagnosed the patient as having HCV-related thrombocytopenia. We could not perform a hepatic biopsy because of the risk for hemorrhage. To suppress HCV replication and improve the thrombocytopenia, treatment with IFN alfa-2a 3 million U TIW was started. We did not observe any adverse effects related to the IFN therapy. At the first visit (during week 2), a mildly elevated thrombocyte count $(15,000$ cells $/ \mathrm{mL})$ was noted. At week 4 , the thrombocyte count was markedly increased (to 123,000 cells $/ \mathrm{mL}$ ), and the ALT level was normalized. However, at week 12 , the platelet count decreased markedly, to 35,000 cells/mL, ALT level was increased to $56 \mathrm{IU} / \mathrm{L}$, and HCV-RNA level remained $>10^{5}$ copies $/ \mathrm{mL}$. Accordingly, we decided to stop standard IFN treatment. Although the combination of pegylated IFN plus ribavirin is the standard protocol for chronic HCV infection, ${ }^{3}$ a literature search (key terms: interferon, thrombocytopenia, and hepatitis C; years: 1980-2005) revealed that few scientific data concerning the treatment of HCV-related thrombocytopenia are available. However, because combination therapy, at least theoretically, is more effective than IFN therapy in suppressing HCV replication, we decided to start combination therapy with pegylated IFN and ribavirin.

At week 12, the HCV-RNA level decreased to $10^{3}$ copies $/ \mathrm{mL}$, ALT and AST levels normalized, and the platelet count increased to 120,000 cells $/ \mathrm{mL}$. At week 24, HCV-RNA level was still $10^{3}$ copies/mL and the platelet count had increased to 198,000 cells $/ \mathrm{mL}$. At week 48 of combination treatment, HCV-RNA was still 
$<10^{5}$ copies $/ \mathrm{mL}$ and the platelet count was 186,000 cells $/ \mathrm{mL}$. We stopped the treatment at week 48 and monitored the patient at 12-week intervals.

At the time of this writing, the patient had completed 12 months of follow-up, at which time the HCV-RNA levels were $<10^{5}$ copies $/ \mathrm{mL}$ and platelet counts were $176,000,195,000,156,000$, and 188,000 cells $/ \mathrm{mL}$ at $3,6,9$, and 12 months, respectively. Although the patient experienced flulike symptoms in the first 4 weeks of treatment, we did not observe any severe adverse effects associated with combination therapy. Because the patient refused to undergo a hepatic biopsy at the end of therapy, histopathologic data were not available. However, clinical and laboratory data strongly excluded hepatic cirrhosis and portal hypertension. Furthermore, the patient did not show any signs of malignant hematologic processes during the follow-up period.

\section{Discussion}

Thrombocytopenia, a common complication of HCV infection, has been observed in $41 \%$ of patients with $\mathrm{HCV}$ (worldwide). ${ }^{1,3} \mathrm{HCV}$-related thrombocytopenia is classified as mild or severe. In patients with HCV infection and thrombocytopenia, mild thrombocytopenia, defined as a thrombocyte count $<150,000$ cells $/ \mathrm{mL}$, is observed in $41 \%$ to $50 \%$ of HCV-infected patients. Severe thrombocytopenia, defined as a count $<50,000$ cells $/ \mathrm{mL}$, is observed in $9 \%$ of patients. ${ }^{3,5} \mathrm{~A}$ recent epidemiologic study ${ }^{6}$ indicated a strong association between low platelet count and $\mathrm{HCV}$ infection but not hepatitis $\mathrm{B}$ virus (HBV) infection. In that study of 1690 patients, thrombocytopenia was found in $10.2 \%$ of patients with HCV infection, $5.2 \%$ of patients coinfected with HBV and HCV, $1.9 \%$ of those with HBV infection, and $1.3 \%$ of control subjects. ${ }^{6}$

In another study, elevated levels of platelet-associated IgG were observed more frequently in patients with chronic HCV infection (88.1\%) than in patients with chronic HBV infection $(47.1 \%)(P<0.05) .{ }^{7}$ In a serial report of $\mathrm{HCV}$-related cytopenias, autoimmune hemolytic anemia and neutropenia were usually related to or associated with other underlying autoimmune phenomena ( $71 \%$ of patients) ${ }^{8}$ Although these patients responded to steroids, they had a poor survival rate. In contrast, patients with $\mathrm{HCV}$-related severe thrombocytopenia had a lower prevalence of associated autoimmune diseases (11\%) and a poorer response to corticosteroids (55\%) but lower mortality (6\%), with some patients having HIV/HBV coinfections.

The etiology of HCV-related thrombocytopenia is obscure. Although immunologic mechanisms, such as antiplatelet antibodies and anticardiolipin antibodies, are present in some patients, the clinical significance of these has not been validated. ${ }^{9}$ A thrombokinetic study revealed a significant, nearly linear, delayed splenic accumulation; normal or low-normal mean platelet life span; low-normal recovery; depressed platelet production; and a normal or slightly elevated megakaryocyte count in the bone marrow. HCV infection of the megakaryocytes has been found in some patients. ${ }^{10}$ Several authors have re- 
ported the presence of HCV-RNA in peripheral platelets, suggesting direct infection of the platelets as the cause of increased destruction. ${ }^{5,11-17}$ However, HCV genotypes have no effect on the severity of thrombocytopenia. ${ }^{11}$ These data appear to rule out immunologic mechanisms.

Another possible factor in the etiology of thrombocytopenia may be depressed synthesis of thrombopoietin. Inadequate thrombopoietin production may contribute to cirrhotic thrombocytopenia. After hepatic transplantation, thrombopoietin production is restored, leading to the resolution of thrombocytopenia.${ }^{18}$ However, this mechanism is related to the severity of hepatic fibrosis, and patients with HCV-related thrombocytopenia typically are not cirrhotic.

Some antibodies may bind to platelet Fc receptor, resulting in the accumulation of immune complexes on platelets and their subsequent destruction. Mixed essential cryoglobulinemia has been reported in $80 \%$ to $90 \%$ of patients with HCV. ${ }^{19}$ The immune response against HCV infection leads to the production of IgM rheumatoid factor, which can bind to HCV-IgG (type 2 cryoglobulinemia). Anticardiolipin antibodies may also bind to platelets, causing platelet destruction. In fact, many other autoantibodies have been reported with $\mathrm{HCV}$ infection. In a recent series, $36 \%$ of patients with ITP were HCV positive, and platelet-associated Igs (PAIgs) were reported in $75 \%$ to $88 \%$ of patients with $\mathrm{HCV}^{20}$ However, 1 study failed to find any correlation between PAIgs and thrombocytopenia observed in HCV infection. ${ }^{21}$ Sanjo et al ${ }^{22}$ found a significant negative correlation between serum PAIg concentration and splenic volume, suggesting that hypersplenism and immune-mediated processes are the predominant mechanisms of thrombocytopenia. However, the patient in the present report had no circulating PAIgs or splenomegaly.

The appropriate treatment of patients with HCV-associated thrombocytopenia is an important unresolved problem, particularly because the pathogenesis of thrombocytopenia is not well established. The various treatment modalitiesincluding steroids, IV Igs, and splenectomy-have failed to achieve lasting responses..$^{2,3,23}$

IFN treatment has been investigated in isolated HCV-related thrombocytopenia in some studies, 2 of which have reported sustained improvement of thrombocytopenia, even in the first weeks of treatment. ${ }^{11,13}$ However, in the present case, thrombocytopenia improved in the first 4 weeks but was subsequently exacerbated. In addition, viral load failed to decrease by $>2$ logs. All of these aspects indicate a breakthrough of HCV infection at week 12. Actually, standard IFNs have a sustained viral response rate of $\sim 20 \%$. The best predictor of IFN nonresponse is the failure of $\mathrm{HCV}$ clearance at week 12 . In the present case, initial improvement and subsequent relapse of thrombocytopenia might have been related to IFN resistance.

The fluctuation in platelet count is not a rare phenomenon in HCV infection. ${ }^{11}$ However, the most common scenario is the progression from HCV infection without thrombocytopenia to HIV with thrombocytopenia. Based on the literature search, the wide range of spontaneous fluctuations in platelet count seen in the 
present case (from 10,000 to $>100,000$ cells $/ \mathrm{mL}$ ) has not been reported. We believe this is clearly a treatment-related improvement of thrombocytopenia.

Pegylated IFN is a long-acting standard IFN that has been shown in large trials to be more effective compared with conventional IFN treatment. ${ }^{24-26}$ The improved response in the present case might have resulted from the sustained suppression of $\mathrm{HCV}$ replication by pegylated IFN plus ribavirin combination therapy.

\section{CONCLUSIONS}

In this case report, combination treatment with pegylated IFN plus ribavirin was effective in the treatment of HCV-related thrombocytopenia. However, further research is needed to define the response rate in different patient populations.

\section{REFERENCES}

1. Gumber SC, Chopra S. Hepatitis C: A multifaceted disease. Review of extrahepatic manifestations. Ann Intern Med. 1995;123:615-620.

2. Durand JM, Cretel E, Retornaz F, et al. Alpha interferon therapy in thrombocytopenia associated with hepatitis C virus infection. J Hepatol. 1994;21:277-279.

3. Linares M, Pastor E, Hemandez F, et al. Autoimmune thrombocytopenia and hepatitis C virus infection. Am J Hematol. 1996;53:284.

4. George JN. Platelets. Lancet. 2000;355:1531-1539.

5. Negamine T, Ohtuka T, Takehara K, et al. Thrombocytopenia associated with hepatitis C viral infection. J Hepatol. 1996;24:135-140.

6. Wang CS, Yao WJ, Wang ST, et al. Strong association of hepatitis C virus (HCV) infection and thrombocytopenia: Implications from a survey of a community with hyperendemic HCV infection. Clin Infect Dis. 2004;39:790-796.

7. Leroy V, Arvieux J, Jacob MC, et al. Prevalence and significance of anticardiolipin, anti-beta2 glycoprotein I and anti-prothrombin antibodies in chronic hepatitis $\mathrm{C}$. Br J Haematol. 1998;101:468-474.

8. Ramos-Casals M, Garcia-Carrasco M, Lopez-Medrano F, et al. Severe autoimmune cytopenias in treatment-naive hepatitis $\mathrm{C}$ virus infection: Clinical description of 35 cases. Medicine (Baltimore). 2003;82:87-96.

9. Hernandez F, Blanquer A, Linares M, et al. Autoimmune thrombocytopenia associated with hepatitis C virus infection. Acta Haematol. 1998;99:217-220.

10. Bordin G, Ballare M, Zigrossi P, et al. A laboratory and thrombokinetic study of HCVassociated thrombocytopenia: A direct role of HCV in bone marrow exhaustion? Clin Exp Rheumatol. 1995;13(Suppl 13):S39-S43.

11. de Almeida AJ, Campos-de-Magalhaes M, de Melo Marcal OP, et al. Hepatitis C virusassociated thrombocytopenia: A controlled prospective, virological study. Ann Hematol. 2004;83:434-440.

12. Silva M, Li X, Cheinquer $\mathrm{H}$, et al. HCV associated idiopathic thrombocytopenic purpura (TTP). Gastroenterology. 1992;102:A889. Abstract.

13. Li XM, Jeffers LJ, Shao LJ, et al. Platelets are carriers of HCV-RNA in patients with HCV infection. Gastroenterology. 1994;106(Suppl):A903. Abstract. 
14. Takehara K, Otsuka T, Arai T, et al. Detection of hepatitis $\mathrm{C}$ virus (HCV) in platelets on type $\mathrm{C}$ chronic liver diseases by polymerase chain reaction (PCR). Gastroenterology. 1994;106(Suppl):A995. Abstract.

15. Floreani A, Biasin MR, Marchelle G, et al. Are human platelets a site of HCV replication? Hepatology. 1996;24:511A. Abstract.

16. Lerat $\mathrm{H}$, Rumin $\mathrm{S}$, Habersetzer $\mathrm{F}$, et al. In vivo tropism of hepatitis $\mathrm{C}$ virus genomic sequences in hematopoietic cells: Influence of viral load, viral genotype, and cell phenotype. Blood. 1998;91:3841-3849.

17. Hamaia S, Li C, Allain JP. The dynamics of hepatitis $C$ virus binding to platelets and 2 mononuclear cell lines. Blood. 2001;98:2293-2300.

18. Goulis J, Chau TN, Jordan S, et al. Thrombopoietin concentrations are low in patients with cirrhosis and thrombocytopenia and are restored after orthotopic liver transplantation. Gut. 1999;44:754-758.

19. Pivetti S, Novarino A, Merico F, et al. High prevalence of autoimmune phenomena in hepatitis $C$ virus antibody positive patients with lymphoproliferative and connective tissue disorders. Br J Haematol. 1996;95:204-211.

20. Kosugi S, Tomiyama Y, Shiraga M, et al. Platelet-associated anti-glycoprotein (GP) IlaIIIb autoantibodies in chronic immune thrombocytopenic purpura mainly recognize cation-dependent conformations: Comparison with the epitopes of serum autoantibodies. Thromb Haemost. 1996;75:339-345.

21. Kosugi S, Imayi Y, Kurata Y, et al. Platelet-associated IgM elevated in patients with chronic hepatitis C contains no platelet autoantibodies. Liver. 1997;17:230-237.

22. Sanjo A, Satoi J, Ohnishi A, et al. Role of elevated platelet-associated immunoglobulin $\mathrm{G}$ and hypersplenism in thrombocytopenia of chronic liver diseases. $J$ Gastroenterol Hepatol. 2003; 18:638-644.

23. Bauduer F, Marty F, Larrouy M, Ducout L. Immunologic thrombocytopenic purpura as presenting symptom of hepatitis C infection. Am J Hematol. 1998;57:338-340.

24. Manns MP, McHutchison JG, Gordon SC, et al. Peginterferon alfa-2b plus ribavirin compared with interferon alfa-2b plus ribavirin for initial treatment of chronic hepatitis C: A randomised trial. Lancet. 2001;358:958-965.

25. Fried MW, Shiffman ML, Reddy KR, et al. Peginterferon alfa-2a plus ribavirin for chronic hepatitis C virus infection. $N$ Engl J Med. 2002;347:975-982.

26. Hadziyannis SJ, Sette H Jr, Morgan TR, et al, for the PEGASYS International Study Group. Peginterferon-alpha2a and ribavirin combination therapy in chronic hepatitis C: A randomized study of treatment duration and ribavirin dose. Ann Intern Med. 2004;140:346-355.

Address correspondence to: Tarkan Karakan, MD, 42 cad 453 sok 1/26 Cukurambar, Ankara, Turkey. E-mail: tkarakan@gazi.edu.tr 